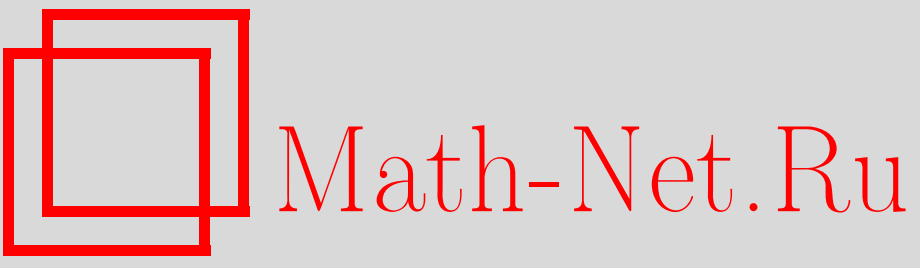

V. N. Ushakov, A. R. Matviichuk, A. V. Ushakov, Approximations of attainability sets and of integral funnels of differential inclusions, Vestn. Udmurtsk. Univ. Mat. Mekh. Komp. Nauki, 2011, Issue 4, 23-39

Use of the all-Russian mathematical portal Math-Net.Ru implies that you have read and agreed to these terms of use

http://www.mathnet.ru/eng/agreement

Download details:

IP : 34.229 .108 .108

April 26, 2023, 11:27:02 


\title{
УДК 517.977.5
}

\author{
(C) В.Н. Уиаков, А.Р. Матвийчук, А.В. Уиаков
}

\section{АППРОКСИМАЦИЯ МНОЖЕСТВ ДОСТИЖИМОСТИ И ИНТЕГРАЛЬНЫХ ВОРОНОК ДИФФЕРЕНЦИАЛЬНЫХ ВКЛЮЧЕНИЙ ${ }^{1}$}

Работа посвящена дифференциальным включениям (д.в.) на конечном промежутке времени. Обсуждаются вопросы, касающиеся вычисления множеств достижимости д.в. Как правило, множества достижимости не поддаются эффективному аналитическому описанию. В то же время часто возникает потребность в их вычислении. Довольно часто она появляется, например, в теории управления, стимулируя развитие методов и алгоритмов приближенного вычисления множеств достижимости.

Ключевые слова: множества достижимости, интегральная воронка, дифференциальное включение.

\section{Введение}

Набор методов и подходов к приближенному вычислению множеств достижимости достаточно богат. В основном они связаны с теми или иными способами подмены управляемой системы или д.в. на д.в. с более простой динамикой. В настоящей работе приводится одна общая схема пошагового приближенного вычисления множеств достижимости д.в. Она сводится фактически к замене конечного промежутка времени, на котором рассматривается д.в., конечным разбиением и к подмене локальных множеств достижимости на промежутках разбиения некоторыми близкими множествами, удобными для вычислений. Корректность такой подмены подтверждается соответствующими оценками - из этих оценок следует, что результаты приближенных вычислений множеств достижимости д.в. сходятся к множествам достижимости д.в. при шаге разбиения, стремящемся к нулю.

Тематика работы примыкает к работам [1-10], в которых исследуются интегральные воронки динамических систем, свойство инвариантности в различных формулировках, а также рассматриваются вопросы, связанные с аппроксимацией множеств достижимости динамических систем.

\section{§1. Дифференциальные включения на конечном промежутке времени}

Пусть на промежутке $\left[t_{0}, \vartheta\right], t_{0}<\vartheta<\infty$ задано д.в.

$$
\frac{d x}{d t} \in F(t, x), \quad x \in \mathbb{R}^{n}
$$

Здесь отображение $(t, x) \mapsto F(t, x)$ удовлетворяет следующим условиям.

A.1. $F(t, x)$ - выпуклый компакт в $\mathbb{R}^{n}$ при $(t, x) \in\left[t_{0}, \vartheta\right] \times \mathbb{R}^{n}$.

A.2. $(t, x) \mapsto F(t, x)$ непрерывно на $\left[t_{0}, \vartheta\right] \times \mathbb{R}^{n}$ в хаусдорфовой метрике: для любой точки $\left(t_{*}, x_{*}\right) \in\left[t_{0}, \vartheta\right] \times \mathbb{R}^{n}$

$$
d\left(F(t, x), F\left(t_{*}, x_{*}\right)\right) \rightarrow 0
$$

при $(t, x) \rightarrow\left(t_{*}, x_{*}\right)\left((t, x) \in\left[t_{0}, \vartheta\right] \times \mathbb{R}^{n}\right)$.

\footnotetext{
${ }^{1}$ Работа выполнена при финансовой поддержке РФФИ (проект 08-01-00587-а), программы государственной поддержки ведущих научных школ (НШ-2640.2008.1), регионального гранта РФФИ/ПСО (проект 07-0196085) и гранта ОФИМ-1 «Методы позиционных дифференциальных игр в задачах техники, экономики и экологии» 11-01-12088.
} 
A.3. $(t, x) \mapsto F(t, x)$ локально липшицево по $x$ : для любой ограниченной и замкнутой области $D \subset\left[t_{0}, \vartheta\right] \times \mathbb{R}^{n}$ найдется такая константа $L=L(D) \in(0, \infty)$, что

$$
d\left(F\left(t, x_{*}\right), F\left(t, x^{*}\right)\right) \leqslant L\left\|x_{*}-x^{*}\right\|
$$

здесь $L=L(D) \in(0, \infty)$ при $\left(t, x_{*}\right)$ и $\left(t, x^{*}\right)$ из $D$.

A.4. Существует такая константа $\gamma \in(0, \infty)$, что выполняется неравенство

$$
\max _{f \in F(t, x)}\|f\| \leqslant \gamma(1+\|x\|)
$$

при $(t, x) \in\left[t_{0}, \vartheta\right] \times \mathbb{R}^{n}$.

Здесь $d\left(F_{*}, F^{*}\right)=\max \left\{h\left(F_{*}, F^{*}\right), h\left(F^{*}, F_{*}\right)\right\}$ - хаусдорфово расстояние между компактами $F_{*}$ и $F^{*}$ из $\mathbb{R}^{n} ; h\left(F_{*}, F^{*}\right)=\max _{f_{*} \in F_{*}} \rho\left(f_{*}, F_{*}\right)$ - хаусдорфово отклонение $F_{*}$ от $F^{*} ; \rho\left(f_{*}, F^{*}\right)=$ $\min _{f^{*} \in F^{*}}\left\|f_{*}-f^{*}\right\| ;$ знак $\rightarrow$ означает сходимость.

Напомним некоторые известные определения из теории дифференциальных включений.

Решением д.в. (1.1) на $\left[t_{0}, \vartheta\right]$ с начальным условием $x\left(t_{0}\right)=x_{0}$ называется абсолютно непрерывная на $\left[t_{0}, \vartheta\right]$ вектор-функция $x=x(t), x\left(t_{0}\right)=x_{0}$, такая, что

$$
\frac{d x(t)}{d t} \in F(t, x(t))
$$

почти всюду (п.в.) на $\left[t_{0}, \vartheta\right]$.

Определение 1. Назовем множеством достижимости $X\left(t^{*}, t_{0}, x_{0}\right)$ д.в. (1.1), отвечающим моменту $t^{*} \in\left[t_{0}, \vartheta\right]$, множество всех таких $x^{*} \in \mathbb{R}^{n}$, что $x^{*}=x\left(t^{*}\right)$ для некоторого решения $x(t), x\left(t_{0}\right)=x_{0}$, д.в. $(1.1)$.

Полагая $X_{0} \subset \mathbb{R}^{n}$, введем обозначение $X\left(t^{*}, t_{0}, X_{0}\right)=\bigcup_{x_{0} \in X_{0}} X\left(t^{*}, t_{0}, x_{0}\right)$ - множество достижимости д.в. (1.1), отвечающее моменту $t^{*}$ и начальному множеству $X_{0}$.

Определение 2. Назовем интегральной воронкой д.в. (1.1) на $\left[t_{0}, \vartheta\right]$ с начальным условием $x\left(t_{0}\right)=x_{0}$ множество $X\left(t_{0}, x_{0}\right)=\bigcup_{t^{*} \in\left[t_{0}, \vartheta\right]}\left(t^{*}, X\left(t^{*}, t_{0}, x_{0}\right)\right) \subset\left[t_{0}, \vartheta\right] \times \mathbb{R}^{n}$.

Здесь $\left(t^{*}, X^{*}\right)=\left\{\left(t^{*}, x^{*}\right): x^{*} \in X^{*}\right\}$.

Полагаем также $X\left(t_{0}, X_{0}\right)=\bigcup_{x_{0} \in X_{0}} X\left(t_{0}, x_{0}\right) \subset\left[t_{0}, \vartheta\right] \times \mathbb{R}^{n}$ - интегральная воронка д.в. (1.1) на $\left[t_{0}, \vartheta\right]$ с начальным множеством $X_{0}$.

Обозначим через comp $\mathbb{R}^{n}$ метрическое пространство, элементы которого - компакты из $\mathbb{R}^{n}$, а метрика - хаусдорфова, то есть $d(\cdot, \cdot)$.

Пусть $X_{0} \in \operatorname{comp} \mathbb{R}^{n}$. Из условий А.1-А.4 следует

$$
X\left(t^{*}\right)=X\left(t^{*}, t_{0}, X_{0}\right) \in \operatorname{comp} \mathbb{R}^{n} \quad \text { при } \quad t^{*} \in\left[t_{0}, \vartheta\right] .
$$

Кроме того, отображение $t^{*} \mapsto X\left(t^{*}\right)$ непрерывно на $\left[t_{0}, \vartheta\right]$ в хаусдорфовой метрике.

Таким образом, отображение $t^{*} \mapsto X\left(t^{*}\right) \in \operatorname{comp} \mathbb{R}^{n}$ представляет собой математический объект, обладающий рядом хороших свойств.

Параллельно с выяснением различных полезных свойств множеств достижимости $X\left(t^{*}\right)$ д.в. (1.1) при рассмотрении конкретных д.в. часто возникает вопрос о (точном) вычислении этих множеств или об их (точном) эффективном аналитическом описании. Здесь встает непростой вопрос о том, что следует понимать под (точным) вычислением множеств $X\left(t^{*}\right)$ и (точным) эффективным аналитическим описанием этих множеств. Под (точным) вычислением множества $X\left(t^{*}\right)$ будем понимать вычисление точек множества $X\left(t^{*}\right)$ с определенной высокой степенью точности за приемлемое время. Под эффективным (точным) аналитическим описанием 
множеств $X\left(t^{*}\right)$ будем понимать представление этих множеств с помощью конечного числа операций пересечения и объединения, примененных к некоторому конечному набору множеств $\Phi_{i_{1} i_{2} \ldots i_{N}}$ в $\mathbb{R}^{n}, i_{k} \in \overline{1, I_{k}}, k=\overline{1, N}$; здесь каждое из множеств $\Phi_{i_{1} i_{2} \ldots i_{N}}$ есть множество Лебега некоторой скалярной функции $\varphi_{i_{1} i_{2} \ldots i_{N}}(x)$, то есть $\Phi_{i_{1} i_{2} \ldots i_{N}}=\left\{x \in \mathbb{R}^{n}: \varphi_{i_{1} i_{2} \ldots i_{N}}(x) \leqslant 0\right\}$, где $\varphi_{i_{1} i_{2} \ldots i_{N}}(x)$ представима в виде суперпозиции конечного набора элементарных функций от $x$.

В качестве простого примера эффективного (точного) аналитического описания множеств $X\left(t^{*}\right)$ укажем, например, такое множество в $\mathbb{R}^{n}:$

$$
X\left(t^{*}\right)=\bigcap_{i_{3} \in \overline{1, I_{3}}} \bigcup_{i_{2} \in \overline{1, I_{2}}} \bigcap_{i_{1} \in \overline{1, I_{1}}} \Phi_{i_{1} i_{2} i_{3}}
$$

где $\Phi_{i_{1} i_{2} i_{3}}=\left\{x \in \mathbb{R}^{n}: \varphi_{i_{1} i_{2} i_{3}}(x) \leqslant 0\right\}, I_{1}=2, I_{2}=3, I_{3}=2, \varphi_{i_{1} i_{2} i_{3}}(x)=\sin \left(\left(i_{1}+i_{2}+i_{3}\right)\|x\|\right)$, $x \in \mathbb{R}^{n}$.

Описание множества $X\left(t^{*}\right)$ в этом примере мы называем эффективным (точным) аналитическим описанием; здесь существенно то, что число операций объединения и пересечения конечно и каждое множество $\Phi_{i_{1} i_{2} i_{3}}$ представимо как множество Лебега некоторой достаточно простой скалярной функции $\varphi_{i_{1} i_{2} i_{3}}(x)$.

Отметим, что (точное) вычисление множества $X\left(t^{*}\right), t^{*} \in\left[t_{0}, \vartheta\right]$ возможно далеко не для всякого д.в. (1.1); далеко не для всякого д.в. (1.1) возможно эффективное (точное) аналитическое описание множеств $X\left(t^{*}\right)$. Поэтому актуальна задача о приближенном вычислении множеств достижимости $X\left(t^{*}\right), t^{*} \in\left[t_{0}, \vartheta\right]$.

\section{$\S 2$. Схема приближенного вычисления множеств достижимости и интегральных воронок д.в. (1.1)}

В этом параграфе приведем одну достаточно общую схему приближенного вычисления множеств достижимости и интегральных воронок д.в. (1.1).

Пусть задано некоторое множество $X_{0} \subset \operatorname{comp} \mathbb{R}^{n}$. Наряду с ним зададим некоторое число $\gamma_{0} \in(0, \infty)$, удовлетворяющее неравенству $h\left(X_{0},\{\mathbf{0}\}\right)<\gamma_{0}$.

Введем в рассмотрение замкнутую и ограниченную область $D=\left\{(t, x): t \in\left[t_{0}, \vartheta\right], x \in\right.$ $B(\mathbf{0} ; \gamma(t))\}$ в $\left[t_{0}, \vartheta\right] \times \mathbb{R}^{n}$, где $\gamma(t)=\left(\gamma_{0}+\gamma\left(t-t_{0}\right)\right) e^{\gamma\left(t-t_{0}\right)}$ при $t \in\left[t_{0}, \vartheta\right]$ и число $\gamma$ определено условием А.4.

Область $D$ есть интегральная воронка д.в. $\dot{x} \in U(x)$ на $\left[t_{0}, \vartheta\right]$ с начальным множеством $B\left(\mathbf{0} ; \gamma_{0}\right)$; здесь $B(\mathbf{0} ; r)$ - замкнутый шар в $\mathbb{R}^{n}$ с центром в $\mathbf{0}$ радиуса $r, U(x)=B(\mathbf{0}, \gamma(1+\|x\|))$, $x \in \mathbb{R}^{n}$.

Из определения области $D$ следует, что для любых $\left(t_{*}, x_{*}\right) \in D,\left[t_{*}, t^{*}\right] \subset\left[t_{0}, \vartheta\right]$ и любого решения $x(t), x\left(t_{*}\right)=x_{*}$, д.в. (1.1) на $\left[t_{*}, t^{*}\right]$ выполняется включение $(t, x(t)) \in D$. При этом с учетом выбора числа $\gamma_{0}$ получаем, что существует такое достаточно малое $\varepsilon^{*}>0$, при котором $X(t)+B\left(\mathbf{0} ; \varepsilon^{*}\right) \subset D(t), \quad t \in\left[t_{0}, \vartheta\right]$. Это включение можно записать в виде

$$
X\left(t_{0}, X_{0}\right)+\left(0, B\left(\mathbf{0} ; \varepsilon^{*}\right)\right) \subset D .
$$

Включение (2.1) означает, что интегральная воронка $X\left(t_{0}, X_{0}\right)$ содержится в $D$ с некоторым запасом. Этот запас нам необходим для того, чтобы в процессе построения множеств, аппроксимирующих интегральную воронку $X\left(t_{0}, X_{0}\right)$, соответствующие аппроксимации не выходили из $D$. Именно эту область $D$ вместе с константой Липшица $L=L(D)$ будем рассматривать в наших построениях; вместе с ней будет встречаться в наших рассуждениях и число

$$
K=\max \{\|f\|: f \in F(t, x),(t, x) \in D\} \in(0, \infty) .
$$

В основу приближенного вычисления множеств достижимости д.в. (1.1) положим полугрупповое свойство этих множеств

$$
X\left(t^{*}\right)=X\left(t^{*}, t_{*}, X\left(t_{*}\right)\right), \quad t \leqslant t_{*}<t^{*} \leqslant \vartheta .
$$


Использование этого свойства вместе с дискретизацией времени (введение конечного разбиения промежутка $\left.\left[t_{0}, \vartheta\right]\right)$ и последующим применением к д.в. (1.1) конструкций в духе ломаных Эйлера (см., например, [5]) выделяют контуры определенного направления в разработке методов приближенного вычисления множеств $X(t)=X\left(t, t_{0}, X_{0}\right), t \in\left[t_{0}, \vartheta\right]$.

Сначала охарактеризуем кратко это направление.

Вводим разбиение $\Gamma=\left\{t_{0}, t_{1}, \ldots, t_{i}, \ldots, t_{N}=\vartheta\right\}$ промежутка $\left[t_{0}, \vartheta\right]$ с равными шагами $\Delta_{i}=$ $t_{i+1}-t_{i}, i=\overline{0, N-1}$, считая при этом, что диаметр $\Delta=\Delta_{i}$ разбиения $\Gamma$ мал.

Если бы вдруг оказалось, что при малых $\delta=t^{*}-t_{*}>0$ мы в состоянии точно вычислять множества $X\left(t^{*}, t_{*}, X_{*}\right), X_{*} \in \operatorname{comp} \mathbb{R}^{n}$, то мы смогли бы для вычисления множеств $X\left(t_{i}\right)$, $t_{i} \in \Gamma$, воспользоваться рекуррентным соотношением

$$
X\left(t_{i}\right)=X\left(t_{i}, t_{i-1}, X\left(t_{i-1}\right)\right), i=\overline{0, N-1} .
$$

Соотношения (2.4) представляют некоторую пошаговую (по времени) конструкцию вычисления множеств достижимости $X\left(t_{i}\right)$. Следует отметить, что стремление к применению пошаговых конструкций в теории динамических систем при вычислении множеств достижимости связано с представлениями о том, что, зная исходное множество $X_{*} \in \operatorname{comp} \mathbb{R}^{n}$, отвечающее некоторому моменту $t_{*}$, и динамику системы, можно достаточно точно спрогнозировать множество достижимости $X\left(t^{*}, t_{*}, X_{*}\right)$, отвечающее моменту $t^{*}>t_{*}$, близкому к $t_{*}$.

Однако осуществить (точное) вычисление даже множеств $X\left(t^{*}, t_{*}, x_{*}\right)$ для сколько-нибудь нетривиальных д.в. мы не можем, что влечет невозможность вычисления и множеств $X\left(t_{i}\right)$, $i=\overline{0, N}$. Учитывая это обстоятельство, мы вынуждены обратиться к разработке методов приближенного вычисления множеств достижимости $X\left(t_{i}\right)$.

Обращение при проведении приближенных вычислений множеств $X\left(t_{i}\right), t_{i} \in \Gamma$ к конструкциям типа ломаных Эйлера означало бы для д.в. (1.1) подмену множеств $X\left(t^{*}, t_{*}, x_{*}\right)$ при малых $\delta=t^{*}-t_{*}>0$ (локальных множеств достижимости д.в. (1.1)) множествами $\widetilde{X}\left(t^{*}, t_{*}, x_{*}\right)=x_{*}+\delta F\left(t_{*}, x_{*}\right)$, где обозначено $x_{*}+\delta F\left(t_{*}, x_{*}\right)=\left\{x_{*}+\delta f_{*}: f_{*} \in F\left(t_{*}, x_{*}\right)\right\}$.

Множества $\widetilde{X}\left(t^{*}, t_{*}, x_{*}\right)$ гораздо более удобны для вычислений, чем множества $X\left(t^{*}, t_{*}, x_{*}\right)$ : они представляют собой выпуклые компакты в $\mathbb{R}^{n}$, гомотетичные множеству $F\left(t_{*}, x_{*}\right)$. Если при этом компакты $F\left(t_{*}, x_{*}\right)$ имеют простую геометрию, например, шары, эллипсоиды или выпуклые многогранники в $\mathbb{R}^{n}$, то множества $\widetilde{X}\left(t^{*}, t_{*}, x_{*}\right)$ допускают точное аналитическое описание, что очень удобно для вычислений.

Положив $\widetilde{X}\left(t^{*}, t_{*}, X_{*}\right)=\bigcup_{x_{*} \in X_{*}} \widetilde{X}\left(t^{*}, t_{*}, x_{*}\right), X_{*} \in \operatorname{comp} \mathbb{R}^{n}$, мы бы стали приближенно вычислять множества $\widetilde{X}\left(t_{i}\right), t_{i} \in \Gamma$ как множества $X_{a}\left(t_{i}\right)=X_{a}\left(t_{i}, t_{0}, X_{0}\right)$ по рекуррентной формуле

$$
X_{a}\left(t_{0}\right)=X_{0}, X_{a}\left(t_{i}\right)=\tilde{X}\left(t_{i}, t_{i-1}, X_{a}\left(t_{i-1}\right)\right), i=\overline{1, N} .
$$

Здесь $a$ - символ аппроксимации.

Можно показать, что

$$
\lim _{\Delta \rightarrow 0} \max _{t_{i} \in \Gamma} d\left(X\left(t_{i}\right), X_{a}\left(t_{i}\right)\right)=0,
$$

и в этом смысле множества $X_{a}\left(t_{i}\right)$ есть хорошие аппроксимации множеств $X\left(t_{i}\right), t_{i} \in \Gamma$.

Однако и множества $X_{a}\left(t_{i}\right), t_{i} \in \Gamma$ мы не в состоянии вычислить (точно), ибо они (в общем случае) несчетны и имеют, как правило, сложную геометрию, не поддающуюся аналитическому описанию.

Таким образом, отображение

$$
\left(t^{*}, t_{*}, X_{*}\right) \mapsto \widetilde{X}\left(t^{*}, t_{*}, X_{*}\right)
$$

само по себе, без каких-либо его коррекций, не поддается точному вычислению и поэтому не применимо для приближенного вычисления множеств $X\left(t_{i}\right), t_{i} \in \Gamma$ в виде множеств $X_{a}\left(t_{i}\right)$.

Следующий шаг заключается в коррекции отображения (2.5): мы подменяем его таким отображением

$$
\left(t^{*}, t_{*}, X_{*}\right) \mapsto X^{(\delta)}\left(t^{*}, t_{*}, X_{*}\right) \in \operatorname{comp} \mathbb{R}^{n}, \quad \delta=t^{*}-t_{*}>0,
$$


которое поддается точному вычислению и при этом порождает последовательность

$$
X^{a}\left(t_{0}\right)=X_{0}^{(\Delta)}, \quad X^{a}\left(t_{i}\right)=X^{(\delta)}\left(t_{i}, t_{i-1}, X^{a}\left(t_{i-1}\right)\right), \quad i=\overline{1, N}
$$

такую, что

$$
\lim _{\Delta \rightarrow 0} \max _{t_{i} \in \Gamma} d\left(X\left(t_{i}\right), X^{a}\left(t_{i}\right)\right)=0
$$

Здесь $X_{0}^{(\Delta)}$ в $(2.7)$ - некоторое конечное множество в $\mathbb{R}^{n}$, удовлетворяющее неравенству $d\left(X_{0}, X_{0}^{(\Delta)}\right) \leqslant \sigma^{*}(\Delta)$, где $\sigma^{*}(\Delta) \downarrow 0$ при $\Delta \downarrow 0$.

Таково эскизное описание той схемы приближенных вычислений множеств $X\left(t_{i}\right), t_{i} \in \Gamma$, которую мы здесь предлагаем. Опишем теперь подробнее отображение (2.6).

Для этого введем сначала отображение $\left(t_{*}, x_{*}\right) \mapsto F^{(\delta)}\left(t_{*}, x_{*}\right)$ на $D$ (зависящее еще и от параметра $\left.\delta=t^{*}-t_{*}\right)$ - некоторую аппроксимацию отображения $\left(t_{*}, x_{*}\right) \mapsto F\left(t_{*}, x_{*}\right)$ на $D$ такую, что

$$
\sup _{\left(t_{*}, x_{*}\right) \in D} d\left(F\left(t_{*}, x_{*}\right), F^{(\delta)}\left(t_{*}, x_{*}\right)\right) \leqslant \varphi^{*}(\delta),
$$

где $\varphi^{*}(\delta)$ - некоторая функция на $(0, \infty), \varphi^{*}(\delta) \downarrow 0$ при $\delta \downarrow 0$. При этом считаем, что при каждом $\left(t_{*}, x_{*}\right) \in D$ множество $F^{(\delta)}\left(t_{*}, x_{*}\right)$ вычислимо (точно) в том смысле, что это или конечное множество точек в $\mathbb{R}^{n}$, или множество в $\mathbb{R}^{n}$, которое может быть описано аналитически при помощи конечного числа неравенств, например, шар, эллипсоид, пересечение конечного числа шаров, эллипсоидов, или выпуклый многогранник в $\mathbb{R}^{n}$ и так далее.

Далее вводим множество

$$
X^{(\delta)}\left(t^{*}, t_{*}, x_{*}\right)=x_{*}+\delta F^{(\delta)}\left(t_{*}, x_{*}\right) \subset \mathbb{R}^{n}, \quad\left(t_{*}, x_{*}\right) \in D, t^{*} \in\left[t_{*}, \vartheta\right] .
$$

Здесь $x_{*}+\delta F^{(\delta)}\left(t_{*}, x_{*}\right)=\left\{x_{*}+\delta f_{*}: f_{*} \in F^{(\delta)}\left(t_{*}, x_{*}\right)\right\}$.

Оценим сверху величину

$$
\sup _{\left(t_{*}, x_{*}\right) \in D} d\left(X\left(t^{*}, t_{*}, x_{*}\right), X^{(\delta)}\left(t^{*}, t_{*}, x_{*}\right)\right) .
$$

Так как по условию А.2 отображение $(t, x) \mapsto F(t, x)$ непрерывно на $\left[t_{0}, \vartheta\right] \times \mathbb{R}^{n}$ (в хаусдорфовой метрике), то оно равномерно непрерывно на $D$. Значит существует такая функция $\omega^{*}(\rho)$ на $(0, \infty)\left(\omega^{*}(\rho) \downarrow 0\right.$ при $\left.\rho \downarrow 0\right)$, что

$$
d\left(F\left(t_{*}, x_{*}\right), F\left(t^{*}, x^{*}\right)\right) \leqslant \omega^{*}\left(\left|t_{*}-t^{*}\right|+\left\|x_{*}-x^{*}\right\|\right) \quad \text { при } \quad\left(t_{*}, x_{*}\right) \text { и }\left(t^{*}, x^{*}\right) \text { из } D .
$$

Докажем неравенство

$$
\sup _{\left(t_{*}, x_{*}\right) \in D} d\left(X\left(t^{*}, t_{*}, x_{*}\right), \widetilde{X}\left(t^{*}, t_{*}, x_{*}\right)\right) \leqslant \omega(\delta) ;
$$

здесь $\omega(\delta)=\delta \omega^{*}((1+K) \delta), \delta \in(0, \infty)$.

Для обоснования (2.12) возьмем произвольные $x^{*} \in X\left(t^{*}, t_{*}, x_{*}\right),\left(t_{*}, x_{*}\right) \in D, t^{*} \in\left[t_{0}, \vartheta\right]$. Существует такое решение $x(t)$ д.в. (1.1) на $\left[t_{*}, t^{*}\right]$, что $x\left(t_{*}\right)=x_{*}$ и $x\left(t^{*}\right)=x^{*}$.

Для него справедливо представление

$$
x(t)=x_{*}+\int_{t_{*}}^{t} f(\tau) d \tau, \quad t \in\left[t_{*}, t^{*}\right],
$$

где $f(\tau) \in F(\tau, x(\tau))$ п.в. на $\left[t_{*}, t^{*}\right]$.

Принимая во внимание $(2.11)$, получаем для решения $x(t)$ на $\left[t_{*}, t^{*}\right]$ неравенство

$$
\begin{gathered}
d\left(F(t, x(t)), F\left(t_{*}, x_{*}\right)\right) \leqslant \omega^{*}\left(\left|t-t_{*}\right|+\left\|x(t)-x_{*}\right\|\right) \leqslant \\
\leqslant \omega^{*}\left(\delta+\delta \max _{\tau \in\left[t_{*}, t^{*}\right]}\|f(\tau)\|\right) \leqslant \omega^{*}((1+K) \delta) .
\end{gathered}
$$


MАТЕМАТИКА

2011. Вып. 4

Из (2.14) вытекает включение при $t \in\left[t_{*}, t^{*}\right]$

$$
f(t) \in F\left(t_{*}, x_{*}\right)+B\left(\mathbf{0} ; \omega^{*}((1+K) \delta)\right),
$$

из которого следует

$$
\frac{1}{\delta} \int_{t_{*}}^{t^{*}} f(t) d t \in F\left(t_{*}, x_{*}\right)+B\left(\mathbf{0} ; \omega^{*}((1+K) \delta)\right) .
$$

Из этого включения, в свою очередь, следует

$$
x\left(t^{*}\right) \in \widetilde{X}\left(t^{*}, t_{*}, x_{*}\right)+B(\mathbf{0} ; \omega(\delta)) .
$$

Так как точка $x^{*}=x\left(t^{*}\right)$ взята произвольно в $X\left(t^{*}, t_{*}, x_{*}\right)$, то

$$
X\left(t^{*}, t_{*}, x_{*}\right) \subset \widetilde{X}\left(t^{*}, t_{*}, x_{*}\right)+B(\mathbf{0} ; \omega(\delta)) .
$$

Докажем теперь включение

$$
\widetilde{X}\left(t^{*}, t_{*}, x_{*}\right) \subset X\left(t^{*}, t_{*}, x_{*}\right)+B(\mathbf{0} ; \omega(\delta)) .
$$

Для этого возьмем произвольную точку $x^{*} \in \widetilde{X}\left(t^{*}, t_{*}, x_{*}\right)$. Точка $x^{*}$ представима в виде

$$
x^{*}=x_{*}+\delta f_{*}, \quad f_{*} \in F\left(t_{*}, x_{*}\right) .
$$

Наряду с решением $x(t)=x_{*}+\left(t-t_{*}\right) f_{*}$ д.в. $\dot{x} \in F\left(t_{*}, x_{*}\right), x\left(t_{*}\right)=x_{*}$ на $\left[t_{*}, t^{*}\right]$ рассмотрим при каждом $k=1,2, \ldots$ разбиение $\Gamma_{k}=\left\{t_{0}^{(k)}=t_{*}, t_{1}^{(k)}, \ldots, t_{N_{k}}^{(k)}=t^{*}\right\}$ промежутка $\left[t_{*}, t^{*}\right]$ (где $\left.\Delta_{j}^{(k)}=t_{j+1}^{(k)}-t_{j}^{(k)}=\mathrm{const}>0, j \in \overline{0, N_{k}-1}\right)$ и ломаную Эйлера $\widetilde{x}^{(k)}(t)$ на $\left[t_{*}, t^{*}\right]$, заданную соотношениями

$$
\widetilde{x}^{(k)}(t)=\widetilde{x}^{(k)}\left(t_{j}^{(k)}\right)+\left(t-t_{j}^{(k)}\right) f_{j}^{(k)} \quad \text { на } \quad\left[t_{j}^{(k)}, t_{j+1}^{(k)}\right], \quad j \in \overline{0, N_{k}-1},
$$

где $t_{j}^{(k)}$ и $t_{j+1}^{(k)}$ из $\Gamma_{k}$ и $f_{j}^{(k)}$ - ближайший вектор в $F\left(t_{j}^{(k)}, \widetilde{x}^{(k)}\left(t_{j}^{(k)}\right)\right)$ к $f_{*}, \widetilde{x}^{(k)}\left(t_{0}^{(k)}\right)=x_{*}$.

Ломаная Эйлера $\widetilde{x}^{(k)}(t)$ на $\left[t_{*}, t^{*}\right]$ удовлетворяет неравенству $\left\|\widetilde{x}^{(k)}(t)-x_{*}\right\| \leqslant K \delta$ и, значит, согласно (2.11), удовлетворяет неравенству

$$
d\left(F\left(t, \widetilde{x}^{(k)}(t)\right), F\left(t_{*}, x_{*}\right)\right) \leqslant \omega^{*}((1+K) \delta), \quad t \in\left[t_{*}, t^{*}\right] .
$$

Так как $f_{*} \in F\left(t_{*}, x_{*}\right)$, то справедливо

$$
f_{*} \in F\left(t, \widetilde{x}^{(k)}(t)\right)+B\left(\mathbf{0} ; \omega^{*}((1+K) \delta)\right), \quad t \in\left[t_{*}, t^{*}\right] .
$$

В частности, в моменты $t_{j}^{(k)} \in \Gamma_{k}$ выполняется включение

$$
f_{*} \in F\left(t_{j}^{(k)}, \widetilde{x}^{(k)}\left(t_{j}^{(k)}\right)\right)+B\left(\mathbf{0} ; \omega^{*}((1+K) \delta)\right) .
$$

Из равенств

$$
x\left(t_{j+1}^{(k)}\right)-\widetilde{x}^{(k)}\left(t_{j+1}^{(k)}\right)=\left(x\left(t_{j}^{(k)}\right)-\widetilde{x}^{(k)}\left(t_{j}^{(k)}\right)\right)+\Delta_{j}^{(k)}\left(f_{*}-f_{j}^{(k)}\right), \quad j=\overline{0, N_{k}-1} .
$$

следуют неравенства

$$
\left\|x\left(t_{j+1}^{(k)}\right)-\widetilde{x}^{(k)}\left(t_{j+1}^{(k)}\right)\right\| \leqslant\left\|x\left(t_{j}^{(k)}\right)-\widetilde{x}^{(k)}\left(t_{j}^{(k)}\right)\right\|+\Delta_{j}^{(k)}\left\|f_{*}-f_{j}^{(k)}\right\|, \quad j=\overline{0, N_{k}-1} .
$$

Из этих рекуррентных неравенств выводим неравенство

$$
\left\|x\left(t_{N_{k}}^{(k)}\right)-\widetilde{x}^{(k)}\left(t_{N_{k}}^{(k)}\right)\right\| \leqslant \sum_{j=0}^{N_{k}-1} \Delta_{j}^{(k)} \omega^{*}((1+K) \delta),
$$


которое запишем в более компактном виде

$$
\left\|x\left(t^{*}\right)-\widetilde{x}^{(k)}\left(t^{*}\right)\right\| \leqslant \omega(\delta) .
$$

Неравенству (2.20) удовлетворяют все ломаные $\widetilde{x}^{(k)}(t)$ на $\left[t_{*}, t^{*}\right]$. Последовательность $\left\{\widetilde{x}^{(k)}(t)\right\}$ равноограничена и равностепенно непрерывна на $\left[t_{*}, t^{*}\right]$. Следовательно, по теореме Арцела (см., например, $\left[11\right.$, с. 110] ), из последовательности $\left\{\widetilde{x}^{(k)}(t)\right\}$ на $\left[t_{*}, t^{*}\right]$ можно выделить равномерно сходящуюся подпоследовательность $\left\{\widetilde{x}^{(n)}(t)\right\}$. Вектор-функция $x^{*}(t)=\lim _{n \rightarrow \infty} \widetilde{x}^{(n)}(t)$ на $\left[t_{*}, t^{*}\right]$ удовлетворяет соотношениям

$$
\dot{x}^{*}(t) \in F\left(t, x^{*}(t)\right) \text { п.в. на }\left[t_{*}, t^{*}\right], \quad\left\|x^{*}\left(t^{*}\right)-x\left(t^{*}\right)\right\| \leqslant \omega(\delta) .
$$

Отсюда следует включение

$$
x^{*}=x\left(t^{*}\right) \in X\left(t^{*}, t_{*}, x_{*}\right)+B(\mathbf{0} ; \omega(\delta)),
$$

из которого следует включение (2.17). Из включений (2.16), (2.17) следует неравенство (2.12).

Далее оценим сверху величину

$$
\sup _{\left(t_{*}, x_{*}\right) \in D} d\left(\tilde{X}\left(t^{*}, t_{*}, x_{*}\right), X^{(\delta)}\left(t^{*}, t_{*}, x_{*}\right)\right) .
$$

Из оценки (2.9), которой стеснено отображение $\left(t_{*}, x_{*}\right) \mapsto F^{(\delta)}\left(t_{*}, x_{*}\right)$, вытекает оценка

$$
\sup _{\left(t_{*}, x_{*}\right) \in D} d\left(\widetilde{X}\left(t^{*}, t_{*}, x_{*}\right), X^{(\delta)}\left(t^{*}, t_{*}, x_{*}\right)\right) \leqslant \varphi(\delta), \quad t^{*} \in\left[t_{*}, \vartheta\right]
$$

здесь $\varphi(\delta)$ - положительная функция на $(0 ; \infty)$, представимая в виде $\varphi(\delta)=\delta \varphi^{*}(\delta)$.

Учитывая (2.12) и (2.21), получаем

$$
\sup _{\left(t_{*}, x_{*}\right) \in D} d\left(X\left(t^{*}, t_{*}, x_{*}\right), X^{(\delta)}\left(t^{*}, t_{*}, x_{*}\right)\right) \leqslant \zeta(\delta), \quad t^{*} \in\left[t_{*}, \vartheta\right]
$$

где $\zeta(\delta)=\delta \zeta^{*}(\delta), \zeta^{*}(\delta)=\varphi^{*}(\delta)+\omega^{*}((1+K) \delta), \delta>0$.

Так как $\zeta^{*}(\delta) \downarrow 0$ при $\delta \downarrow 0$, то $\zeta(\delta)$ - бесконечно малая величина по сравнению с $\delta \downarrow 0$.

Определим отображение (2.6) равенством

$$
\begin{gathered}
X^{(\delta)}\left(t^{*}, t_{*}, X_{*}\right)=\bigcup_{\bar{x}_{*} \in X_{*}^{\varepsilon(\delta)}} X^{(\delta)}\left(t^{*}, t_{*}, \bar{x}_{*}\right), \\
t_{0} \leqslant t_{*}<t^{*} \leqslant \vartheta, X_{*} \in \operatorname{comp} \mathbb{R}^{n}, \quad \delta=t^{*}-t_{*}>0 .
\end{gathered}
$$

Считаем, что в $(2.23)$ множество $X_{*}^{\varepsilon(\delta)}$ есть конечная $\varepsilon(\delta)$-сеть множества $X_{*}$ в $\mathbb{R}^{n}$, такая что $d\left(X_{*}, X_{*}^{\varepsilon(\delta)}\right) \leqslant \varepsilon(\delta)$; здесь функция $\varepsilon(\delta)$ выбрана одной и той же для всех $X_{*} \in \operatorname{comp} \mathbb{R}^{n}$ и удовлетворяющей соотношению $\delta^{-1} \varepsilon(\delta) \downarrow 0$ при $\delta \downarrow 0$.

При этом считаем также, что если $X_{*}$ конечно, то $X_{*}^{\varepsilon(\delta)}=X_{*}$ и $\varepsilon(\delta) \equiv 0$ на $(0, \infty)$.

Так определенное отображение (2.6) приемлемо для (точных) вычислений в том смысле, что множество $X^{(\delta)}\left(t^{*}, t_{*}, X_{*}\right)$ есть или конечный набор точек в $\mathbb{R}^{n}$ (вычислимый, стало быть, за конечное число операций) или конечный набор множеств в $\mathbb{R}^{n}$, каждое из которых описывается аналитически при помощи конечного числа неравенств. Это обстоятельство с учетом того, что выполняется оценка (2.22), делает отображение (2.6) приемлемым для приближенного вычисления множеств $X\left(t^{*}, t_{*}, X_{*}\right), X_{*} \in \operatorname{comp} \mathbb{R}^{n}$.

Оценим сверху величину

$$
\begin{gathered}
d\left(X\left(t^{*}, t_{*}, X_{*}\right), X^{(\delta)}\left(t^{*}, t_{*}, X_{*}\right)\right), \\
t_{0} \leqslant t_{*}<t^{*} \leqslant \vartheta, \quad X_{*} \in \operatorname{comp} \mathbb{R}^{n},
\end{gathered}
$$


используя при этом оценку $(2.22)$ и определение множества $X_{*}^{\varepsilon(\delta)}$.

Оценим сначала величину $h\left(X\left(t^{*}, t_{*}, X_{*}\right), X^{(\delta)}\left(t^{*}, t_{*}, X_{*}\right)\right)$.

Возьмем произвольную точку $x_{*} \in X_{*}$. Для нее найдется точка $\bar{x}_{*} \in X_{*}^{\varepsilon(\delta)}$ такая, что $\| x_{*}-$ $\bar{x}_{*} \| \leqslant \varepsilon(\delta)$. Наряду с множеством $X\left(t^{*}, t_{*}, x_{*}\right)$ рассмотрим множество $X\left(t^{*}, t_{*}, \bar{x}_{*}\right)$. Принимая во внимание условие А.3 (см. стр. 24) и неравенство $\left\|x_{*}-\bar{x}_{*}\right\| \leqslant \varepsilon(\delta)$, получаем

$$
d\left(X\left(t^{*}, t_{*}, x_{*}\right), X\left(t^{*}, t_{*}, \bar{x}_{*}\right)\right) \leqslant e^{L \delta}\left\|x_{*}-\bar{x}_{*}\right\| \leqslant e^{L \delta} \varepsilon(\delta) .
$$

Кроме того, согласно (2.22), имеем

$$
d\left(X\left(t^{*}, t_{*}, \bar{x}_{*}\right), X^{(\delta)}\left(t^{*}, t_{*}, \bar{x}_{*}\right)\right) \leqslant \zeta(\delta) .
$$

Из оценок (2.25) и (2.26) следует оценка

$$
d\left(X\left(t^{*}, t_{*}, x_{*}\right), X^{(\delta)}\left(t^{*}, t_{*}, \bar{x}_{*}\right)\right) \leqslant e^{L \delta} \varepsilon(\delta)+\zeta(\delta) .
$$

Из этой оценки следует

$$
h\left(X\left(t^{*}, t_{*}, x_{*}\right), X^{(\delta)}\left(t^{*}, t_{*}, \bar{x}_{*}\right)\right) \leqslant e^{L \delta} \varepsilon(\delta)+\zeta(\delta) .
$$

Из (2.27) получаем

$$
h\left(X\left(t^{*}, t_{*}, X_{*}\right), X^{(\delta)}\left(t^{*}, t_{*}, X_{*}\right)\right) \leqslant e^{L \delta} \varepsilon(\delta)+\zeta(\delta) .
$$

Докажем также оценку

$$
h\left(X^{(\delta)}\left(t^{*}, t_{*}, X^{*}\right), X\left(t^{*}, t_{*}, X_{*}\right)\right) \leqslant e^{L \delta} \varepsilon(\delta)+\zeta(\delta) .
$$

В самом деле, пусть $\bar{x}_{*} \in X_{*}^{\varepsilon(\delta)}$. Тогда по определению $\varepsilon(\delta)$-сети $X_{*}^{\varepsilon(\delta)}$, в множестве $X_{*}$ найдется точка $x_{*}$, для которой $\left\|x_{*}-\bar{x}_{*}\right\| \leqslant \varepsilon(\delta)$. Для точек $x_{*}$ и $\bar{x}_{*}$ выполняется неравенство (2.25) и, значит, - неравенство

$$
d\left(X^{(\delta)}\left(t^{*}, t_{*}, \bar{x}_{*}\right), X\left(t^{*}, t_{*}, x_{*}\right)\right) \leqslant e^{L \delta} \varepsilon(\delta)+\zeta(\delta),
$$

из которого следует

$$
h\left(X^{(\delta)}\left(t^{*}, t_{*}, \bar{x}_{*}\right), X\left(t^{*}, t_{*}, x_{*}\right)\right) \leqslant e^{L \delta} \varepsilon(\delta)+\zeta(\delta) .
$$

Из (2.30) следует оценка (2.29).

Из (2.28) и (2.30) выводим

$$
d\left(X\left(t^{*}, t_{*}, X_{*}\right), X^{(\delta)}\left(t^{*}, t_{*}, X^{*}\right)\right) \leqslant e^{L \delta} \varepsilon(\delta)+\zeta(\delta)
$$

при $t_{*}, t^{*}\left(t_{0} \leqslant t_{*}<t^{*} \leqslant \vartheta\right)$ и $X_{*} \in \operatorname{comp} \mathbb{R}^{n}$.

Оценка $(2.31)$ - базовая при выводе оценки для величины $d\left(X\left(t_{i}\right), X^{a}\left(t_{i}\right)\right), t_{i} \in \Gamma$.

Приступим к выводу оценки величины $d\left(X\left(t_{i}\right), X^{a}\left(t_{i}\right)\right)$.

В момент $t_{0}$ имеем $d\left(X\left(t_{0}\right), X^{a}\left(t_{0}\right)\right)=d\left(X_{0}, X_{0}^{(\Delta)}\right) \leqslant \sigma^{*}(\Delta)$.

Пусть в момент $t_{i} \in \Gamma$ вычислено множество $X^{a}\left(t_{i}\right)$. Тогда, согласно $(2.31)$, имеем

$$
\begin{gathered}
d\left(X\left(t_{i+1}, t_{i}, X\left(t_{i}\right)\right), X^{(\delta)}\left(t_{i+1}, t_{i}, X^{a}\left(t_{i}\right)\right)\right) \leqslant \\
\leqslant d\left(X\left(t_{i+1}, t_{i}, X\left(t_{i}\right)\right), X\left(t_{i+1}, t_{i}, X^{a}\left(t_{i}\right)\right)\right)+d\left(X\left(t_{i+1}, t_{i}, X^{a}\left(t_{i}\right)\right), X^{(\delta)}\left(t_{i+1}, t_{i}, X^{a}\left(t_{i}\right)\right)\right) \leqslant \\
\leqslant e^{L \Delta_{i}} d\left(X\left(t_{i}\right), X^{a}\left(t_{i}\right)\right)+e^{L \Delta_{i}} \varepsilon\left(\Delta_{i}\right)+\zeta\left(\Delta_{i}\right) .
\end{gathered}
$$


Это неравенство запишем в виде

$$
d\left(X\left(t_{i+1}\right), X^{a}\left(t_{i+1}\right)\right) \leqslant e^{L \Delta_{i}} d\left(X\left(t_{i}\right), X^{a}\left(t_{i}\right)\right)+e^{L \Delta_{i}} \varepsilon\left(\Delta_{i}\right)+\zeta\left(\Delta_{i}\right), \quad i=\overline{0, N-1} .
$$

Из (2.32) выводим для произвольного $i=\overline{1, N}$

$$
d\left(X\left(t_{i}\right), X^{a}\left(t_{i}\right)\right) \leqslant e^{L \sum_{j=0}^{i-1} \Delta_{j}} d\left(X_{0}, X_{0}^{(\Delta)}\right)+e^{L \sum_{j=0}^{i-1} \Delta_{j}} \sum_{j=0}^{i-1}\left(\varepsilon\left(\Delta_{j}\right)+\zeta\left(\Delta_{j}\right)\right) .
$$

Введем величину $\varepsilon^{*}(\delta)=\delta^{-1} \varepsilon(\delta), \delta \in(0, \infty)$. Имеем $\varepsilon^{*}(\delta) \downarrow 0$ при $\delta \downarrow 0$ (см. стр. 29). Из (2.33) следует оценка

$$
d\left(X\left(t_{i}\right), X^{a}\left(t_{i}\right)\right) \leqslant e^{L\left(t_{i}-t_{0}\right)}\left\{\sigma^{*}(\Delta)+\left(t_{i}-t_{0}\right)\left(\varepsilon^{*}(\Delta)+\zeta^{*}(\Delta)\right)\right\}, \quad i=\overline{1, N} .
$$

Из оценки (2.34) следует предельное соотношение (2.8).

Видим, что скорость стремления к нулю величины $\max _{t_{i} \in \Gamma} d\left(X\left(t_{i}\right), X^{a}\left(t_{i}\right)\right)$ при $\Delta \rightarrow 0$ определяется порядком по $\Delta$ величин $\sigma^{*}(\Delta), \varepsilon^{*}(\Delta)$ и $\zeta^{*}(\Delta)$ при $\Delta \downarrow 0$.

В случае, когда правая часть дифференциального включения (1.1) не зависит от времени $t$ явно (то есть $F(t, x)=F(x))$, вместо модуля непрерывности $\omega^{*}\left(\left|t_{*}-t^{*}\right|+\left\|x_{*}-x^{*}\right\|\right)$ отображения $(t, x) \mapsto F(t, x)$ можем взять функцию $\omega^{*}\left(\left\|x_{*}-x^{*}\right\|\right)$, не зависящую от $t$ и определенную равенством

$$
\omega^{*}(\delta)=L \delta, \quad \delta \in(0, \infty) .
$$

Тогда в неравенстве $(2.34)$ вместо функции $\omega^{*}((1+K) \Delta)$, входящей слагаемым в $\zeta^{*}(\Delta)$ (см. стр. 29), будет стоять функция $\omega^{*}(K \Delta)=L K \Delta, \Delta>0$.

В этом случае неравенство (2.33) примет вид

$$
d\left(X\left(t_{i}\right), X^{a}\left(t_{i}\right)\right) \leqslant e^{L\left(t_{i}-t_{0}\right)}\left\{\sigma^{*}(\Delta)+\left(t_{i}-t_{0}\right)\left(\varepsilon^{*}(\Delta)+\varphi^{*}(\Delta)+L K \Delta\right)\right\}, \Delta>0, t_{i} \in \Gamma .
$$

Функции $\varepsilon^{*}(\delta), \varphi^{*}(\delta)$ и $\sigma^{*}(\delta)$, согласно их определению, мы выбираем из условия их монотонной сходимости к нулю при $\delta \downarrow 0$.

Так, например, если мы положим $\varepsilon^{*}(\delta)=\gamma_{1} \delta^{\frac{1}{2}}, \varphi^{*}(\delta)=\gamma_{2} \delta^{\frac{1}{2}}, \sigma^{*}(\delta)=\gamma_{3} \delta^{\frac{1}{2}}$, то неравенство примет вид (2.35)

$$
d\left(X\left(t_{i}\right), X^{a}\left(t_{i}\right)\right) \leqslant e^{L\left(t_{i}-t_{0}\right)}\left\{\left(\left(t_{i}-t_{0}\right)\left(\gamma_{1}+\gamma_{2}\right)+\gamma_{3}\right) \Delta^{\frac{1}{2}}+L K \Delta\right\}, \quad \Delta>0, \quad t_{i} \in \Gamma .
$$

Следовательно, в этом случае порядок стремления к нулю величины $\max _{t_{i} \in \Gamma} d\left(X\left(t_{i}\right), X^{a}\left(t_{i}\right)\right)$ при $\Delta \downarrow 0$ не меньше, чем $\frac{1}{2}$.

Если же мы положим $\varepsilon^{*}(\delta)=\gamma_{1} \delta, \varphi^{*}(\delta)=\gamma_{2} \delta, \sigma^{*}(\delta)=\gamma_{3} \delta$, то неравенство (2.35) примет вид

$$
d\left(X\left(t_{i}\right), X^{a}\left(t_{i}\right)\right) \leqslant e^{L\left(t_{i}-t_{0}\right)}\left\{\left(\left(t_{i}-t_{0}\right)\left(\gamma_{1}+\gamma_{2}\right)+\gamma_{3}\right)+L K\right\} \Delta, \Delta>0, t_{i} \in \Gamma .
$$

Следовательно, в этом случае порядок стремления к нулю величины $\max _{t_{i} \in \Gamma} d\left(X\left(t_{i}\right), X^{a}\left(t_{i}\right)\right)$ при $\Delta \downarrow 0$ не меньше, чем 1.

Теперь отметим, что в случае конечнозначного отображения $x_{*} \mapsto F^{(\delta)}\left(x_{*}\right)$ на $D$, все множества $X^{a}\left(t_{i}\right), i=\overline{1, N-1}$ конечны. В этом случае множество $X^{a}\left(t_{i}\right)$ совпадает со своей конечной $\varepsilon^{*}(\delta)$-сетью $\left(\varepsilon^{*}(\delta)=0\right.$ на $\left.(0, \infty)\right)$, то есть в этом случае в неравенстве $(2.35)$ функция $\varepsilon_{*}(\Delta)$ отсутствует.

Далее введем в рассмотрение множество $X_{\Gamma}^{a}\left(t_{0}, X_{0}\right)=\bigcup_{t_{i} \in \Gamma}\left(t_{i}, X^{a}\left(t_{i}\right)\right)$, содержащееся в $D$. Это множество имеет непустые (по времени) сечения только в моменты $t_{i} \in \Gamma$.

Затронем вопрос об аппроксимации интегральной воронки $X\left(t_{0}, X_{0}\right)$ - множества в $D \subset$ $\left[t_{0}, \vartheta\right] \times \mathbb{R}^{n}$ множествами $X_{\Gamma}^{a}\left(t_{0}, X_{0}\right)$. При этом расстояние между множествами $X\left(t_{0}, X_{0}\right)$ и 


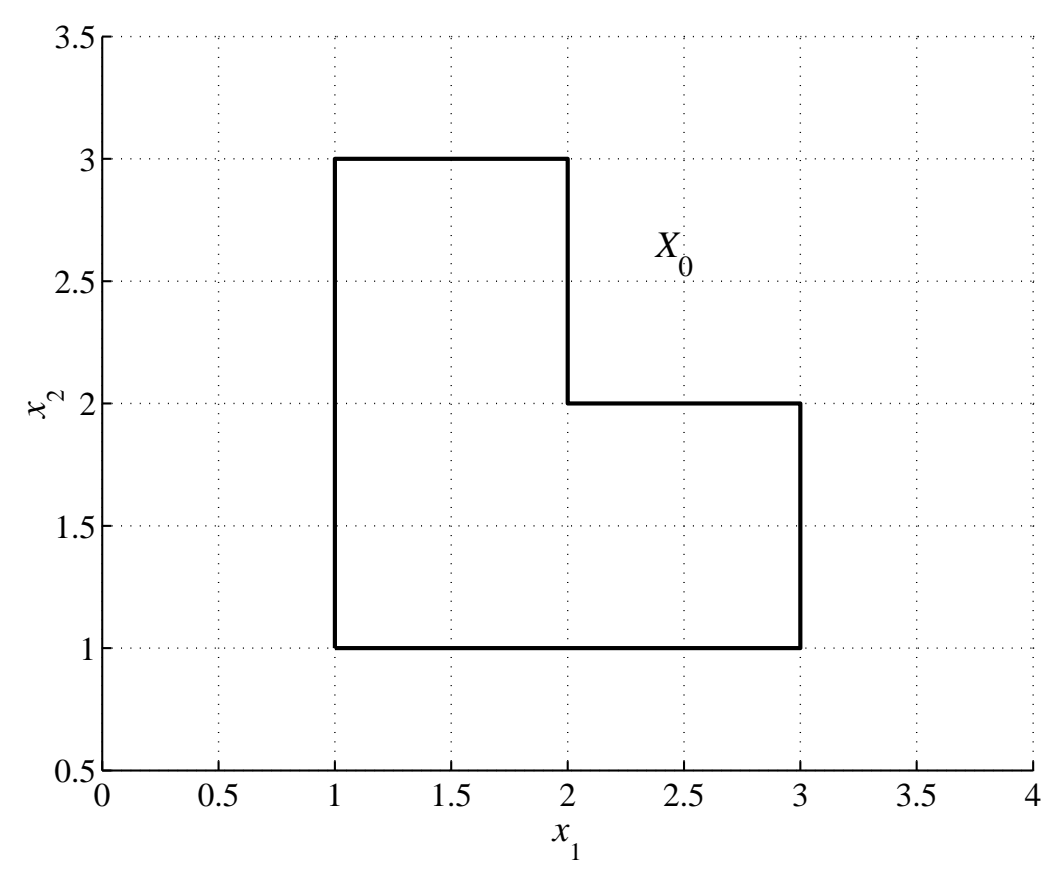

Рис. 1. Множество $X_{0}$

$X_{\Gamma}^{a}\left(t_{0}, X_{0}\right)$ мы измеряем в метрике пространства comp $\mathbb{R}^{n+1}$. Это расстояние так же, как и в случае пространства comp $\mathbb{R}^{n}$, будем обозначать при помощи символа $d(\cdot, \cdot)$.

Из (2.34) следует оценка

$$
d\left(X_{\Gamma}\left(t_{0}, X_{0}\right), X_{\Gamma}^{a}\left(t_{0}, X_{0}\right)\right) \leqslant e^{L\left(\vartheta-t_{0}\right)}\left\{\sigma^{*}(\Delta)+\left(\vartheta-t_{0}\right)\left(\varepsilon^{*}(\Delta)+\zeta^{*}(\Delta)\right)\right\}
$$

здесь $X_{\Gamma}\left(t_{0}, X_{0}\right)=\bigcup_{t_{i} \in \Gamma}\left(t_{i}, X\left(t_{i}\right)\right)$.

Кроме того, для любого промежутка $\left[t_{i}, t_{i+1}\right]$ разбиения $Г$ и любого $t \in\left[t_{i}, t_{i+1}\right]$ справедлива оценка

$$
d\left((t, X(t)),\left(t_{i}, X\left(t_{i}\right)\right)\right) \leqslant(1+K) \Delta
$$

Из (2.38) следует

$$
d\left(X\left(t_{0}, X_{0}\right), X_{\Gamma}\left(t_{0}, X_{0}\right)\right) \leqslant(1+K) \Delta .
$$

Принимая во внимание оценки (2.38), (2.40), получаем

$$
d\left(X\left(t_{0}, X_{0}\right), X_{\Gamma}^{a}\left(t_{0}, X_{0}\right)\right) \leqslant e^{L\left(\vartheta-t_{0}\right)}\left\{\sigma^{*}(\Delta)+\left(\vartheta-t_{0}\right)\left(\varepsilon^{*}(\Delta)+\zeta^{*}(\Delta)\right)\right\}+(1+K) \Delta .
$$

Из оценки (2.41) следует, что

$$
d\left(X\left(t_{0}, X_{0}\right), X_{\Gamma}^{a}\left(t_{0}, X_{0}\right)\right) \rightarrow 0 \text { при } \Delta \rightarrow 0 .
$$

\section{§ 3. Примеры аппроксимации множеств достижимости дифференциальных включений (управляемых систем)}

Рассматриваются две управляемые системы на плоскости на конечном промежутке времени $\left[t_{0}, \vartheta\right]$. Начальное множество (множество начальных точек $x_{0} \in \mathbb{R}^{2}$ ) для этих систем одно и то же: $X_{0}$ есть множество на плоскости $\mathbb{R}^{2}$ (см. рис. 1$)$. 


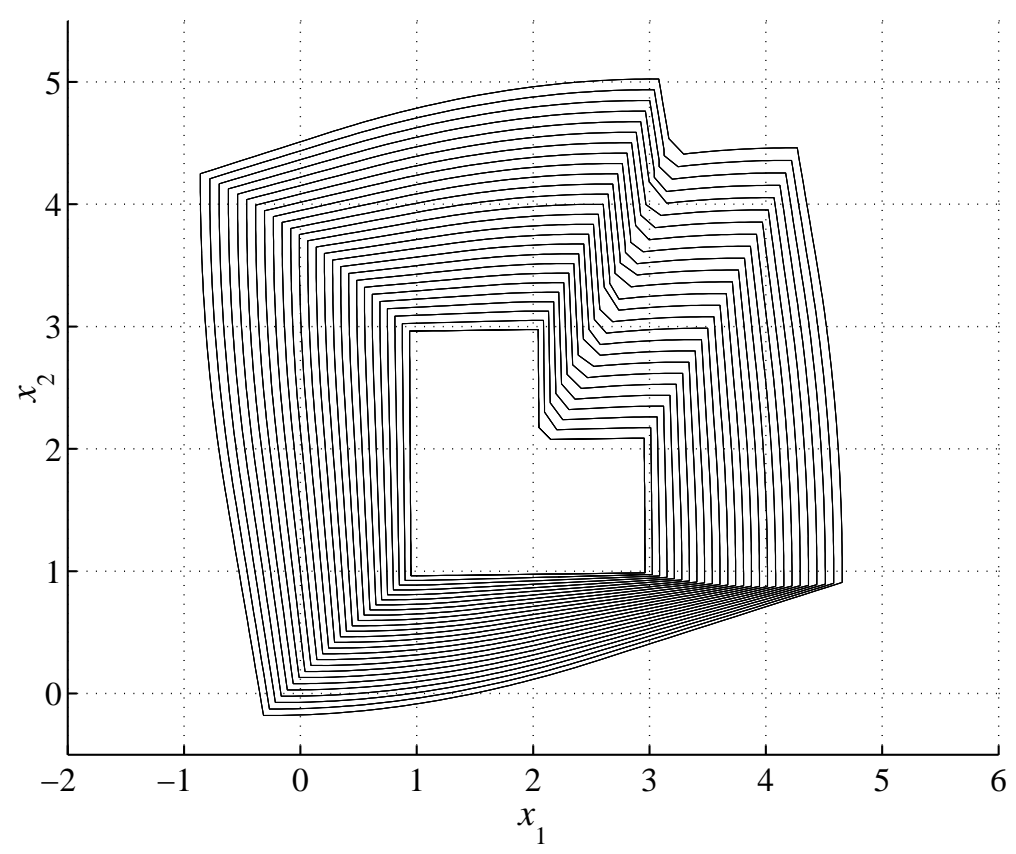

Рис. 2. Множества $X\left(t_{i}\right), t_{i} \in \Gamma$

Пример 1. Задана линейная управляемая система $\Sigma_{1}$ вида

$$
\left\{\begin{array}{l}
\dot{x}_{1}=\frac{1}{8} x_{1}-\frac{1}{8} x_{2}+u_{1}, \\
\dot{x}_{2}=\frac{1}{4} x_{1}+u_{2} ;
\end{array}\right.
$$

здесь $u=\left(u_{1}, u_{2}\right) \in P=\left\{u \in \mathbb{R}^{2}: \max _{i=1,2}\left|u_{i}\right| \leqslant 1\right\}$.

Для системы $\Sigma_{1}$ на промежутке времени $[0,1.2]$ были вычислены приближённо множества достижимости $X\left(t_{i}\right)=X\left(t_{i}, t_{0}, X_{0}\right)$, отвечающие моментам $t_{i}$ разбиения $\Gamma$, имеющего диаметр $\Delta=0.05$. В основу вычислений была положена схема приближенного вычисления множеств достижимости из параграфа 2. Эти множества $X\left(t_{i}\right)$ представлены на рис. 2. Отметим, что в этом простом случае линейной управляемой системы границы $\partial X\left(t_{i}\right)$ множеств $X\left(t_{i}\right)$ выглядят как достаточно регулярные (кусочно-гладкие с небольшим числом кусков гладкости) кривые на плоскости $\mathbb{R}^{2}$.

На рис. 3 представлен в трехмерном пространстве переменных $t, x$ набор множеств $\left\{\left(t_{i}\right.\right.$, $\left.\left.X\left(t_{i}\right)\right)\right\}$, представляющий собой некоторую аппроксимацию интегральной воронки $X\left(t_{0}, X_{0}\right)$ системы $\Sigma_{1}$.

В следующем примере представлена более сложная управляемая система на плоскости $\mathbb{R}^{2}$; она нелинейна по фазовой переменной $x$.

Пример 2. Задана нелинейная управляемая система $\Sigma_{2}$ вида

$$
\left\{\begin{array}{l}
\dot{x}_{1}=-\frac{1}{2} \frac{x_{2}}{\|x\|}+\frac{3}{\|x\|}\left(\sin x_{1} \cdot \sin 2 x_{2}\right)+u_{1}, \\
\dot{x}_{2}=\frac{1}{2} \frac{x_{1}}{\|x\|}+\frac{3}{\|x\|}\left(\sin 2 x_{1} \cdot \sin x_{2}\right) x_{2}+u_{2} ;
\end{array}\right.
$$

здесь $u=\left(u_{1}, u_{2}\right) \in P=\left\{u \in \mathbb{R}^{2}: \max _{i=1,2}\left|u_{i}\right| \leqslant 1\right\}$.

Для системы $\Sigma_{2}$ на промежутке времени $[0,3]$ были вычислены приближенно множества достижимости $X\left(t_{i}\right)=X\left(t_{i}, t_{0}, X_{0}\right)$, отвечающие моментам $t_{i}$ разбиения $\Gamma$, имеющего диаметр 


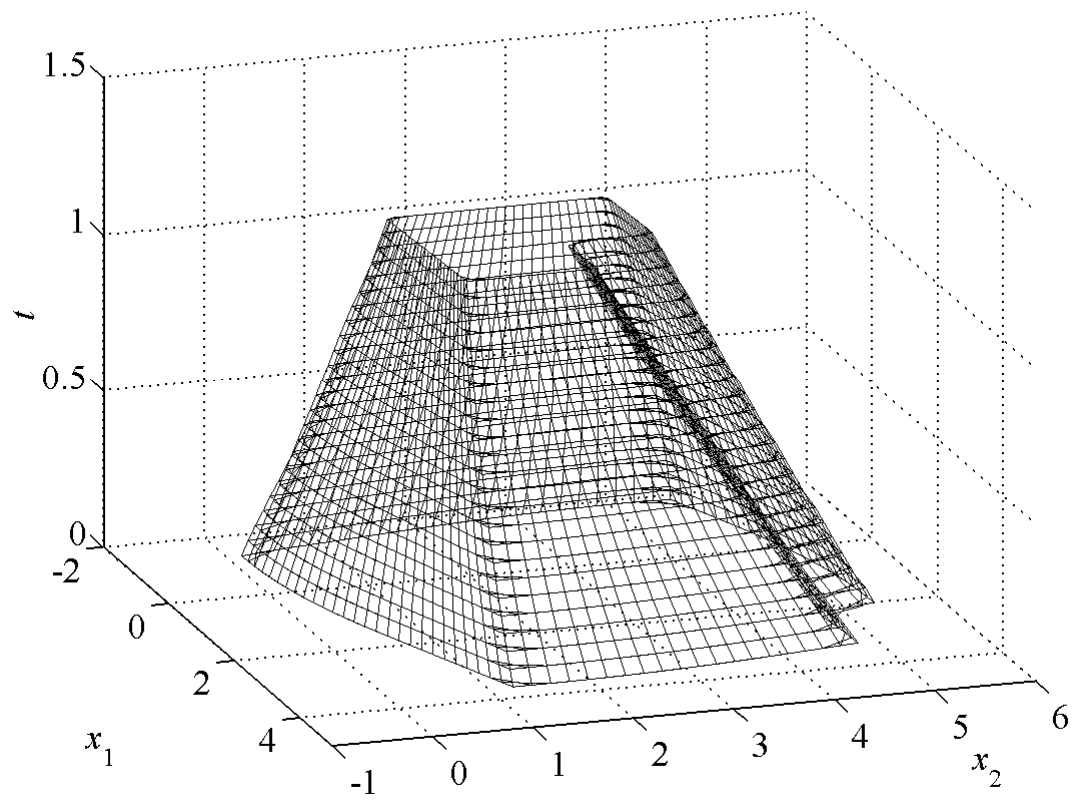

Рис. 3. Множество $\left\{\left(t_{i}, X\left(t_{i}\right)\right)\right\}$

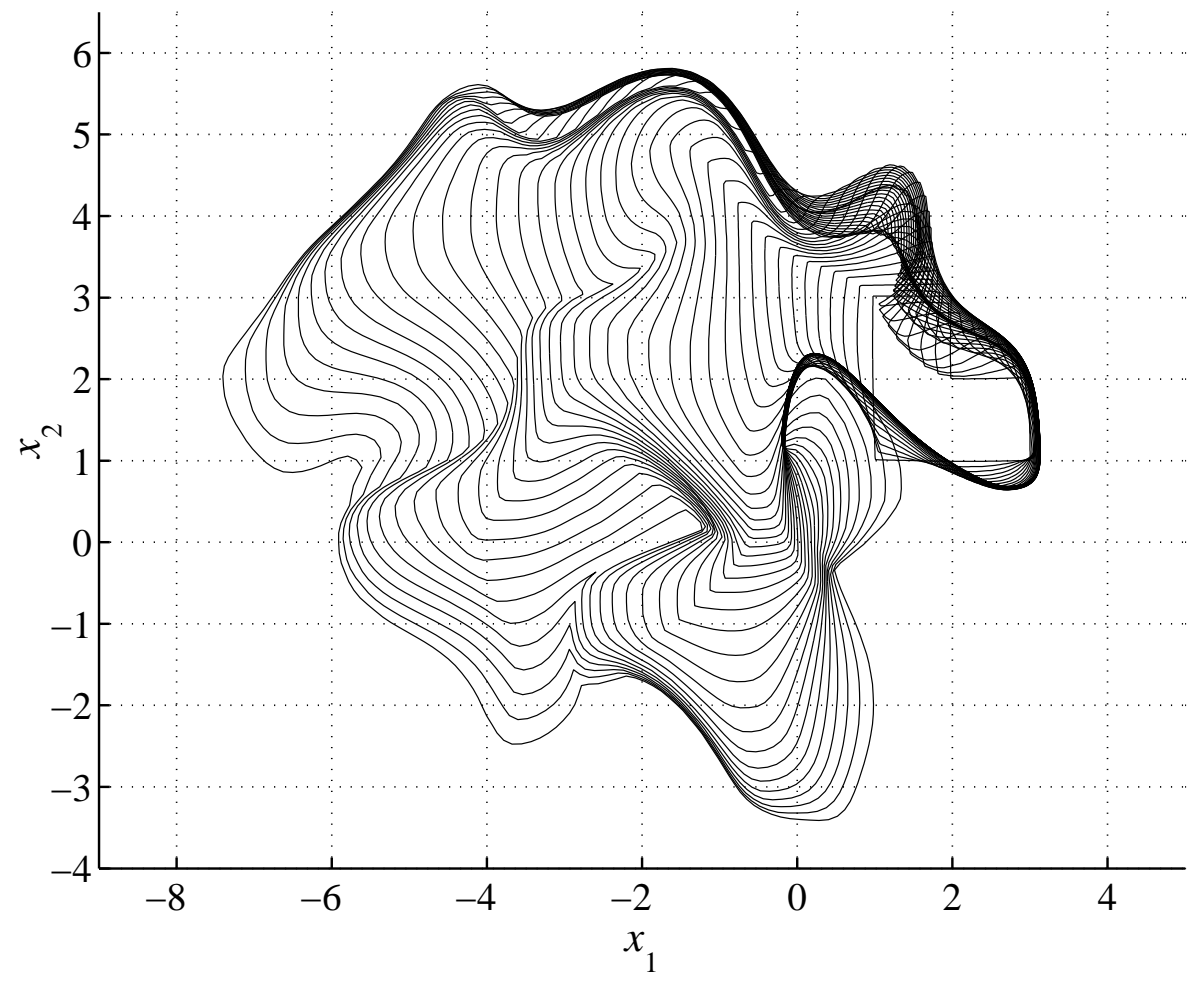

Рис. 4. Множества $X\left(t_{i}\right), t_{i} \in \Gamma$ 


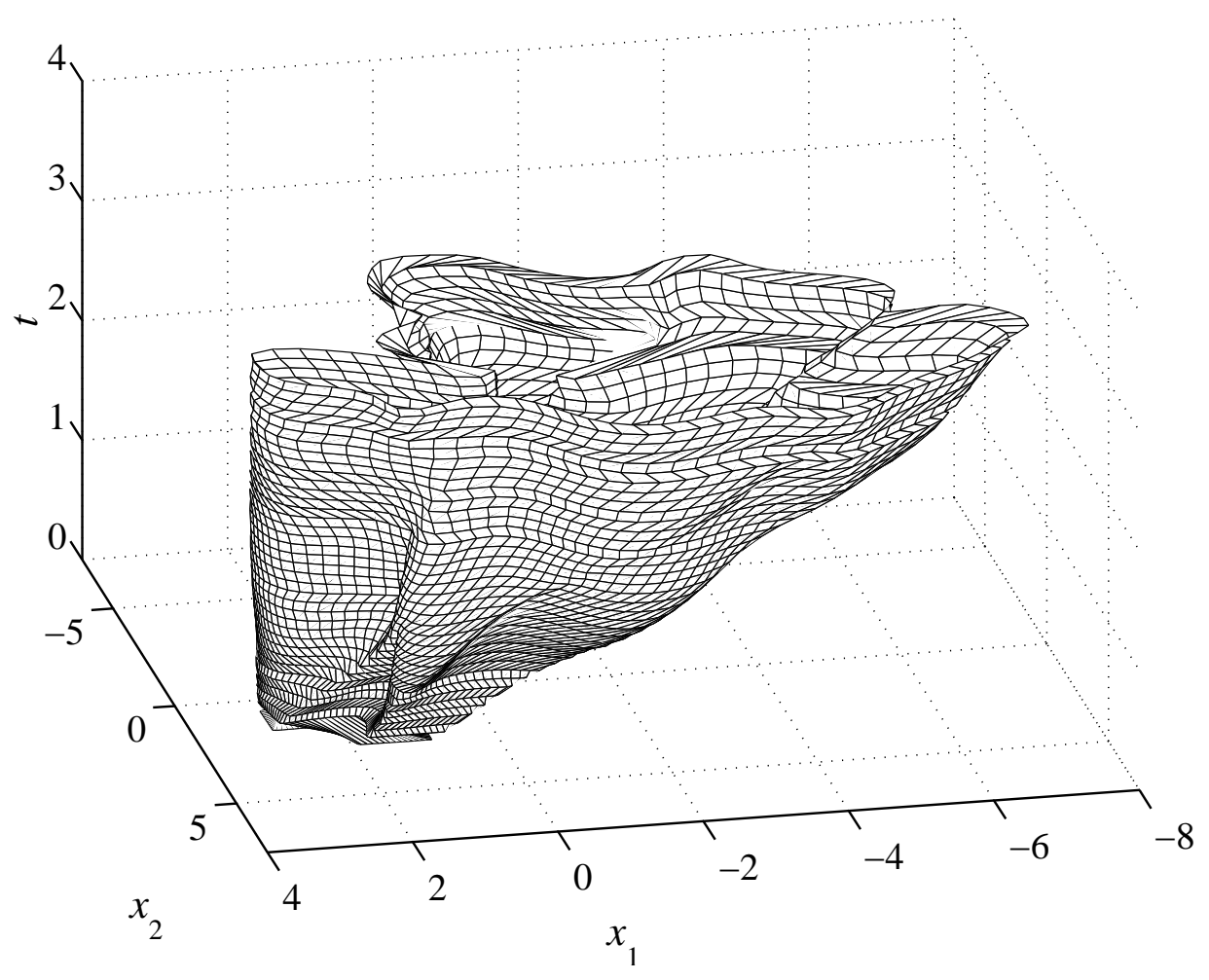

Рис. 5. Множество $\left\{\left(t_{i}, X\left(t_{i}\right)\right)\right\}$

$\Delta=0.01$. Здесь множества достижимости вычислялись на разбиении $\Gamma$, отвечающем более мелкому шагу, чем в предыдущем примере, поскольку система $\Sigma_{2}$ имеет более изощренную динамику. Результаты вычислений изображены на рис. 4-7. При этом на рис. 4 представлен (неполный) набор множеств достижимости $X\left(t_{i}\right)=X\left(t_{i}, t_{0}, X_{0}\right)$ системы $\Sigma_{2}$, идущих с промежутком $\Delta=0.05$ по времени. На рис. 5-7 этот же набор $\left\{X\left(t_{i}\right)\right\}$ представлен в пространстве переменных $t, x$ как набор $\left\{\left(t_{i}, X\left(t_{i}\right)\right)\right\}$ в различных ракурсах. Набор $\left\{\left(t_{i}, X\left(t_{i}\right)\right)\right\}$ представляет собой некоторое приближение интегральной воронки $X\left(t_{0}, X_{0}\right)$ системы $\Sigma_{2}$. В так называемом «обратном» времени $\tau\left(\tau=t+\vartheta-t, t \in\left[t_{0}, \vartheta\right]\right)$ этот набор представляет собой некоторую аппроксимацию множества разрешимости в задаче о сближении системы $\Sigma_{2}^{*}$

$$
\left\{\begin{array}{l}
\dot{x}_{1}=\frac{1}{2} \frac{x_{2}}{\|x\|}-\frac{3}{\|x\|}\left(\sin x_{1} \cdot \sin 2 x_{2}\right)+u_{1}, \\
\dot{x}_{2}=-\frac{1}{2} \frac{x_{1}}{\|x\|}-\frac{3}{\|x\|}\left(\sin 2 x_{1} \cdot \sin x_{2}\right) x_{2}+u_{2},
\end{array}\right.
$$

$u \in P$, с целевым множеством $X_{0}$ в момент $\vartheta=3$ (см. рис. 8-10).

Из анализа геометрической структуры множества разрешимости системы $\Sigma_{2}^{*}$ видно, что построение разрешающих процедур управления, например, процедуры управления с поводырем, идущим по множеству разрешимости, есть непростая задача. В связи с этим обстоятельством имеет смысл при решении задачи о сближении управляемой системы $\Sigma_{2}^{*}$ с целевым множеством $X_{0}$ пытаться сначала огрубить систему $\Sigma_{2}^{*}$ в разумных пределах. То есть имеет смысл сначала огрубить систему $\Sigma_{2}^{*}$ так, чтобы множество разрешимости огрубленной системы было более регулярным и в то же время не слишком отличалось от предыдущего множества разрешимости. Но это также непростая задача. Другой путь состоит в огрублении множества разрешимости (по сечениям $\left(t_{i}, X\left(t_{i}\right)\right)$ этого множества) с последующим использованием этих множеств для построения разрешающих процедур управления. Такой подход представлен, например, в работе [8], посвященной решению игровой задачи о сближении с целевым множеством. 


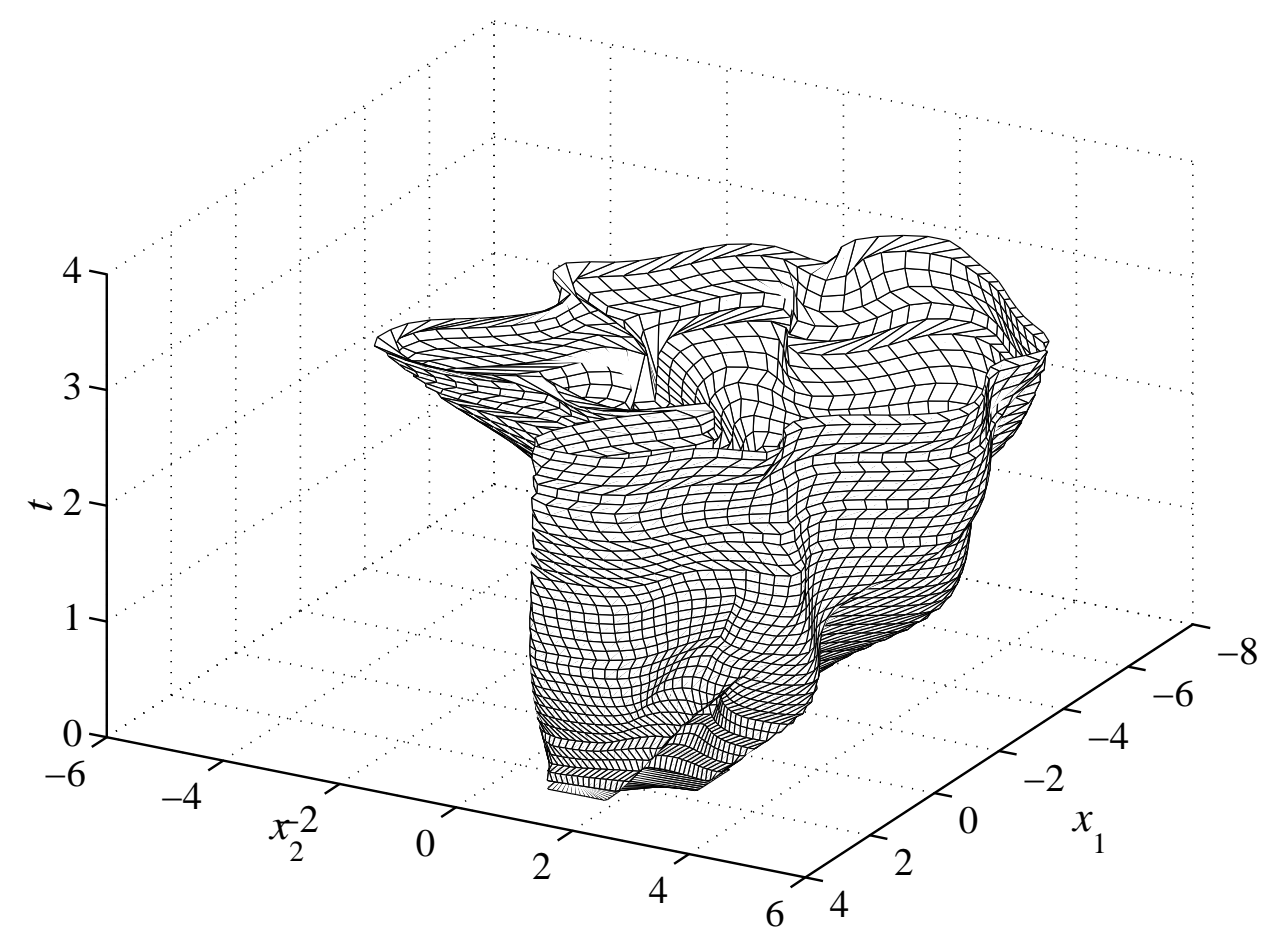

Рис. 6. Множество $\left\{\left(t_{i}, X\left(t_{i}\right)\right)\right\}$

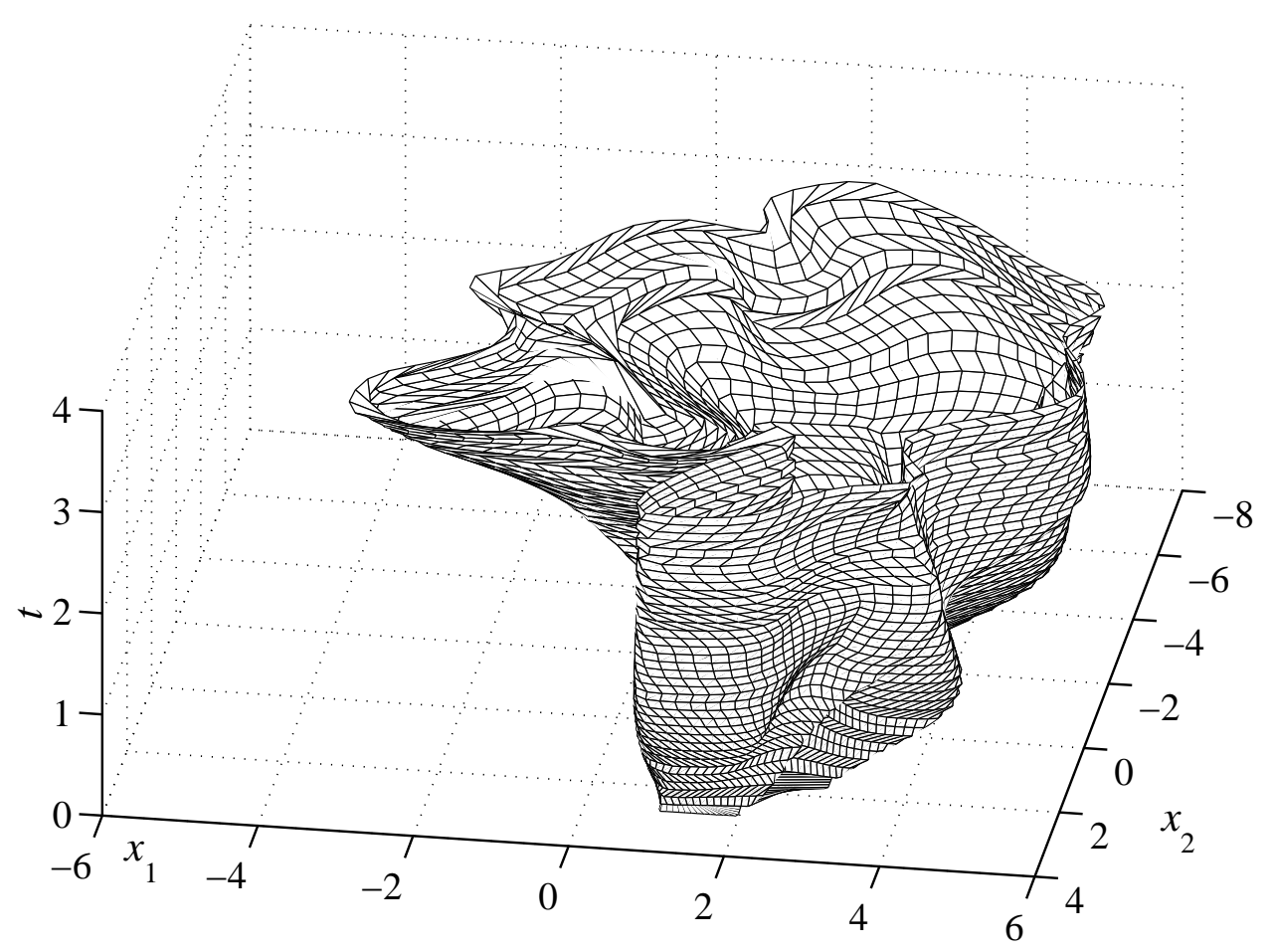

Рис. 7. Множество $\left\{\left(t_{i}, X\left(t_{i}\right)\right)\right\}$ 


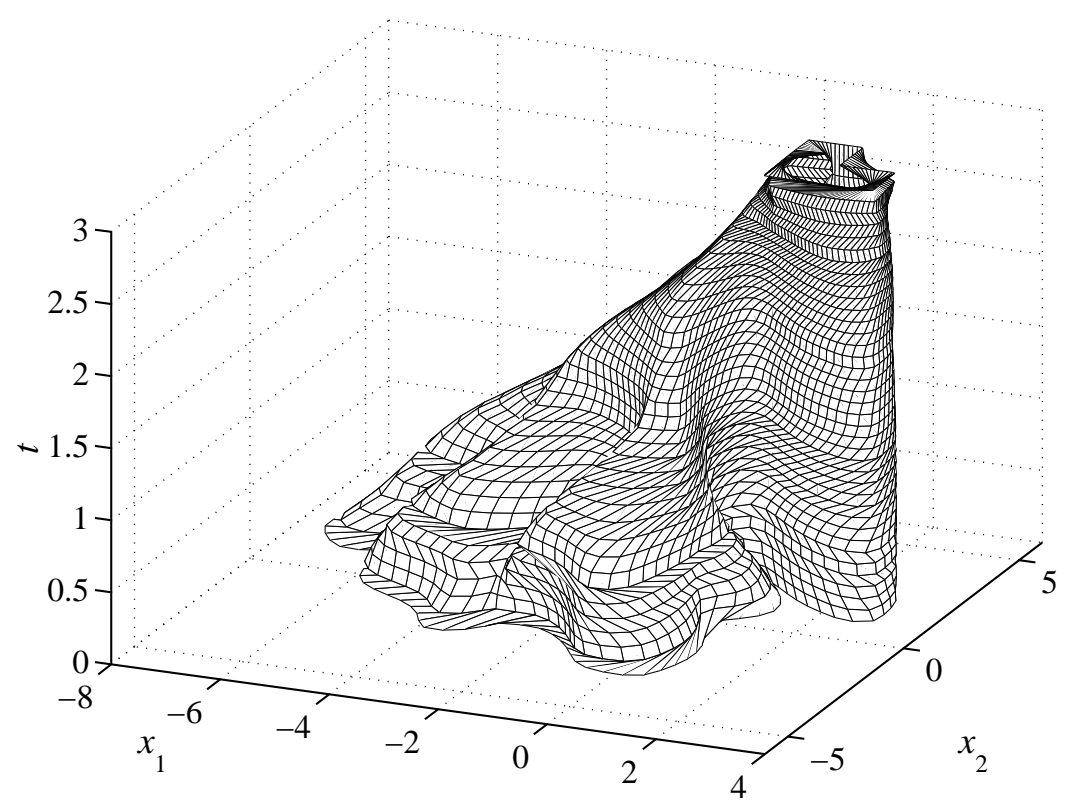

Рис. 8. Множество $\left\{\left(t_{i}, X\left(t_{i}\right)\right)\right\}$ в «обратном» времени

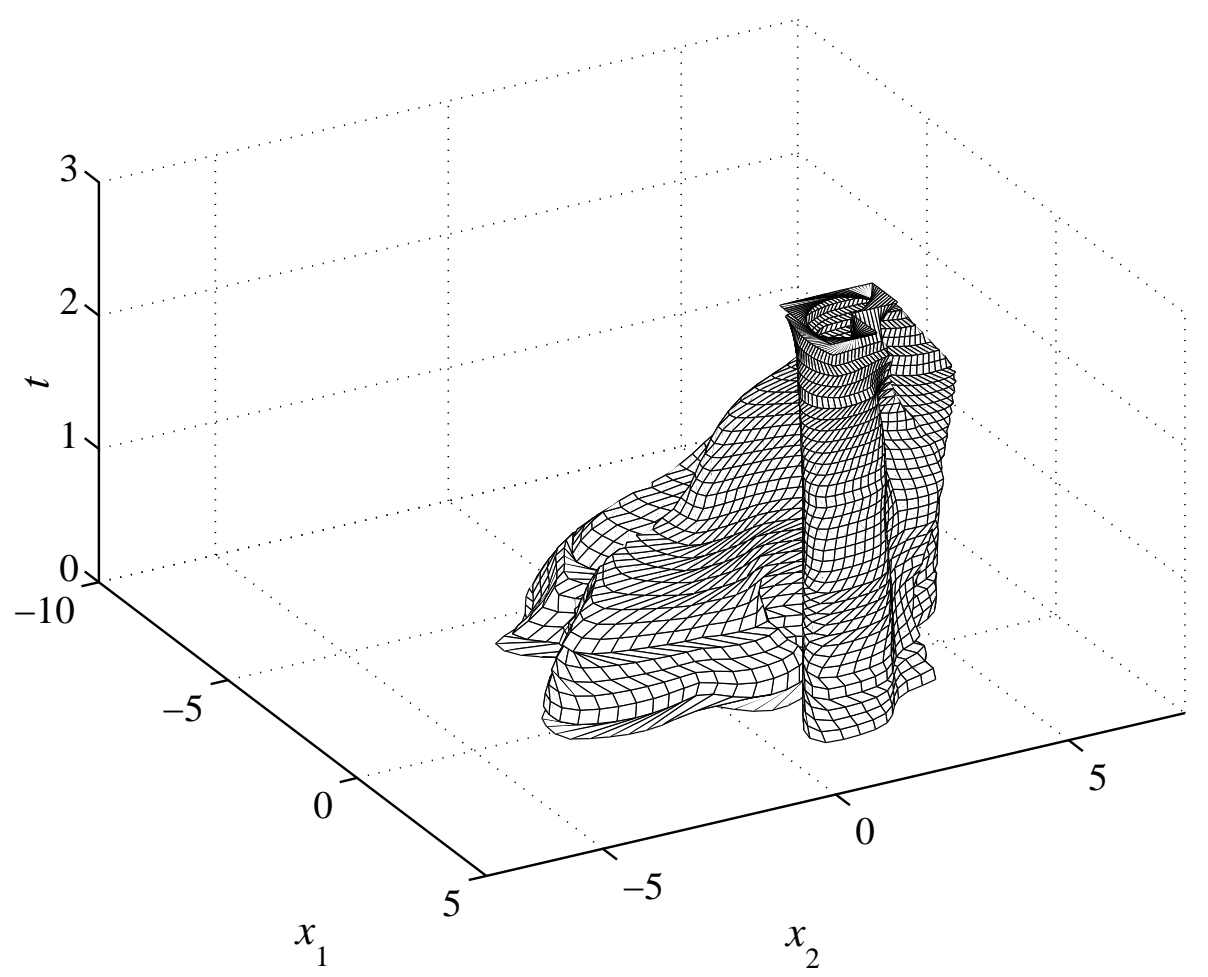

Рис. 9. Множество $\left\{\left(t_{i}, X\left(t_{i}\right)\right)\right\}$ в «обратном» времени 


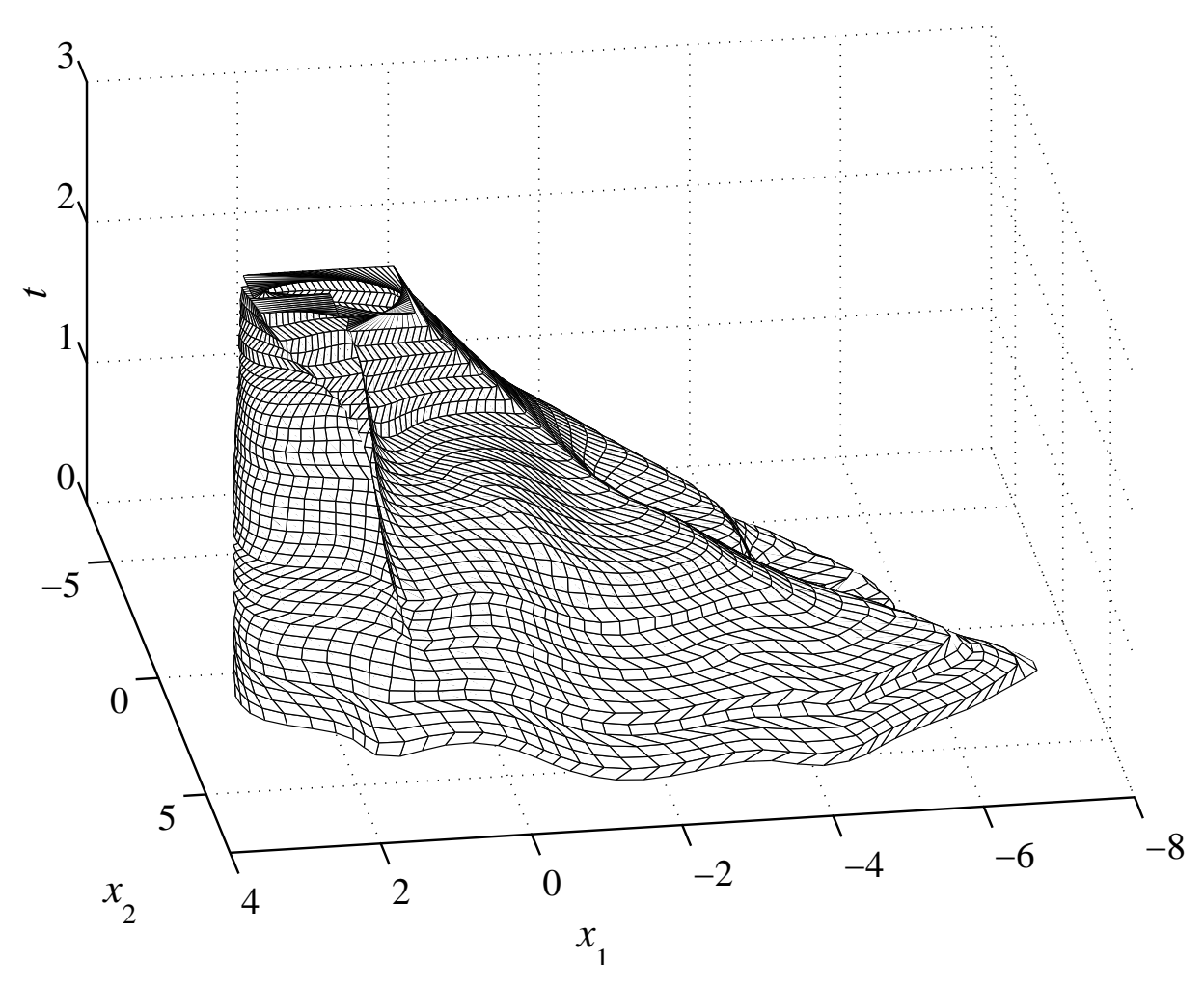

Рис. 10. Множество $\left\{\left(t_{i}, X\left(t_{i}\right)\right)\right\}$ в «обратном» времени

\section{СПИСОК ЛИТЕРАТУРЫ}

1. Красовский Н. Н. Теория управления движением. М.: Наука, 1968. 475 с.

2. Kurzhanski A. B., Valyi I. Ellipsoidal calculus for estimation and control. Boston: Birkhauser, 1997. 321 p.

3. Варайа П., Куржанский А. Б. Эллипсоидальные методы для задач динамики и управления. Ч. 1 // Современная математика и ее приложения. Тбилиси: Ин-т кибернетики АН Грузии, 2005. Т. 23. C. $34-72$.

4. Гусев М. И. Оценки множеств достижимости многомерных управляемых систем с перекрестными связями // Труды Ин-та математики и механики УрО РАН. 2009. Т. 15. № 4. С. 82-94.

5. Гусейнов Х. Г., Моисеев А.Н., Ушаков В.Н. Об аппроксимации областей достижимости управляемых систем // Прикладная матем. и механика. 1998. Т. 62. № 2. С. 179-187.

6. Тонков Е. Л., Панасенко Е. А. Инвариантные и устойчиво инвариантные множества дифференциальных включений // Труды Матем. ин-та им. В.А. Стеклова. 2008. Т. 262. С. 202-221.

7. Родина Л. И., Тонков Е. Л. Статистические характеристики множества достижимости управляемой системы, неблуждаемость и минимальный центр притяжения // Нелинейная динамика. 2009. Т. 5. № 2. C. 265-288.

8. Ушаков В. Н., Матвийчук А. Р., Лебедев П. Д. Дефект стабильности в игровой задаче о сближении в момент // Вестник Удмуртского университета. Математика. Механика. Компьютерные Науки. 2010. Вып. 3. С. 87-103.

9. Филиппова Т. Ф. Построение многозначных оценок множеств достижимости некоторых нелинейных динамических систем с импульсным управлением // Труды Ин-та математики и механики УрО PAH. 2009. T. 15. № 4. C. 263-269.

10. Костоусова Е. К. Об ограниченности и неограниченности внешних полиэдральных оценок множеств достижимости линейных дифференциальных систем // Труды Ин-та математики и механики УрО PAH. 2009. T. 15. № 4. C. 134-145.

11. Колмогоров А.Н., Фомин С. В. Элементы теории функций и функционального анализа. М.: Наука, $1976.544 \mathrm{c}$. 


\section{V.N. Ushakov, A.R. Matviichuk, A. V. Ushakov \\ Approximations of attainability sets and of integral funnels of differential inclusions}

The paper is devoted to differential inclusions (DI) on finite time intervals. We consider some questions of computation of attainable sets for DI. The above sets are rarely describable analytically in an effective way though, often, there is a necessity for their computation, for example, in control theory, in which the above computation is a stimulus to develop methods and algorithms used to approximately compute attainable sets

Keywords: attainability sets, integral funnel, differential inclusion.

Mathematical Subject Classifications: 35F15, 37G10

Ушаков Владимир Николаевич, член-корреспондент РАН, ИММ УрО РАН, 620219, Россия, г. Екатеринбург, ул. С. Ковалевской, 16.

E-mail: ushak@imm.uran.ru

Матвийчук Александр Ростиславович, к. ф.-м. н., научный сотрудник, ИММ УрО РАН, 620219, Россия, г. Екатеринбург, ул. С. Ковалевской, 16.

E-mail: matv@uran.ru

Ушаков Андрей Владимирович, аспирант, ИММ УрО РАН, 620219, Россия, г. Екатеринбург, ул. С. Ковалевской, 16.

E-mail: aushakov.pk@gmail.com

Ushakov Vladimir Nikolaevich, Corresponding Member of RAS, Institute of Mathematics and Mechanics, Ural Branch of the Russian Academy of Sciences, S. Kovalevskaja street, 16, Yekaterinburg, 620990, Russia.

Matviichuk Aleksandr Rostislavovich, Candidate of Physics and Mathematics, Researcher, Institute of Mathematics and Mechanics, Ural Branch of the Russian Academy of Sciences, S. Kovalevskaja street, 16, Yekaterinburg, 620990, Russia.

Ushakov Andrei Vladimirovich, post-graduate student, Institute of Mathematics and Mechanics, Ural Branch of the Russian Academy of Sciences, S. Kovalevskaja street, 16, Yekaterinburg, 620990, Russia. 